\title{
On characterization of probability distributions by means of independent statistics.
}

\author{
IGNaCY X. Kotcarskr, Oklahoma State University (U.S.A.) (*)
}

\begin{abstract}
Summary. - The probability density functions $f_{k}\left(x_{k}\right)=\left.A_{k_{i}} x_{k}\right|_{k} ^{p_{k}-1} e^{-a \psi_{k}\left(x_{k}\right)}$ of independent random variables $x_{0}, x_{1}, \ldots, x_{n}$, are characterized by independence of two functions of them.
\end{abstract}

TaMHANKAR [3] gave a characterization of the normal distribution symmetric about the origin by means of independent statistics. KoTLaRski [2] characterized the gamma distribution in a similar way. In this paper the same method is used to characterize other distributions, where the preceeding two eases are particular ones.

Let $x_{0}, x_{1}, \ldots, x_{n}(n \geq 1)$ be independeut real random variables (r.v.s) having probability density functions (p.d.f.-s) $f_{k}$ satisfying the following conditions:

(A $f_{k}(x)>0$ for $x \in \mathfrak{A}_{k}$, where $\mathfrak{A}_{k}=(-\infty, 0)$ or $(0,+\infty)$

or $(-\infty, 0) \cup(0,+\infty) ; f_{k}(x)=0$ for $x \notin \mathfrak{U}_{k}$;

if $\mathfrak{G}_{k}=(-\infty, 0) \cup(0,+\infty)$ then $f_{k}$ is even;

$f_{k}$ is continuous on $\mathfrak{A}_{k}$;

$\left(\mathrm{A}_{2}\right)$ there exist real numbers $p_{k}$ such that the following limits exist

$$
\lim _{x \rightarrow 0} \frac{f_{k}^{\prime}(x)}{|x|^{p_{k}}-1}=A_{k}>0
$$

Denote the following transformation

(2)

$$
\left\{\begin{array}{c}
x_{0}=y \cdot h_{0}\left(y_{1}, \ldots, y_{n}\right) \\
x_{1}=y \cdot h_{1}\left(y_{1}, \ldots, y_{n}\right) \\
\cdot \cdot \cdot \cdot \cdot \cdot \\
\cdot \cdot \cdot \cdot \cdot \cdot \\
x_{n}=y \cdot h_{n}\left(y_{1}, \ldots, y_{n}\right)
\end{array}\right.
$$

(*) Entrata in Redazione il 12 aprile 1969. 
for $x_{k} \in \mathfrak{A}_{k}, k=0,1 . \ldots, n, y \in \mathfrak{B}_{0}=(0, \infty),\left(y_{1}, \ldots, y_{n}\right) \in \mathfrak{B}$, where $\mathfrak{B}$ is a set in the $n$-dimensional space. Assume that the functions $h_{k}$ on $\mathscr{B}$ are taken in such a way, that there is possible the change of variables in $(n+1)$-dimensional integrals, particularly that the JACOBIAN of (2) exists and does not vanish for $y \in \mathfrak{B}_{0},\left(y_{1}, \ldots, y_{n}\right) \in \mathscr{B}$.

Assume further that there exist functions $\Psi(y), y \in \mathscr{B}_{0}$ transforming $\mathscr{B}_{0}$ onto $\mathfrak{C}=(0, \infty)$ and $\Psi_{k}(\mathfrak{x}), x \in \mathfrak{A}_{k}, k=0,1, \ldots, n$ transforming $\mathfrak{A}_{k}$ onto $\mathfrak{C}$, satisfying the following conditions:

$\left(B_{1}\right) \Psi$ is strictly increasing on $\mathfrak{B}_{0}$, if $(-\infty, 0) \subset \mathfrak{A}_{k}$ then $\Psi_{k}$ is decreasing on it, if $(0, \infty) \subset \mathfrak{Q}_{k}$ then $\Psi_{k}$ is increasing on it, if $\mathfrak{A}_{k}=(-\infty, 0) \cup(0, \infty)$ then $\Psi_{k}$ is even;

$\left(\mathrm{B}_{2}\right)$ there exist limits

$$
\lim _{y \rightarrow 0} \Psi(y)=0, \quad \lim _{x \rightarrow 0} \Psi_{k}(x)=0
$$

$\left(\mathrm{B}_{3}\right) \quad \Psi(y)=\Psi_{0}\left(x_{0}\right)+\Psi_{1}\left(x_{1}\right)+\cdots+\Psi_{n}\left(x_{n}\right)$,

$$
x_{k} \in \mathfrak{A}_{k}, k=0,1, \ldots, n, y \in \mathfrak{B}_{0} \text {; }
$$

(B) the functions

$$
f_{k}(x)= \begin{cases}A_{k}|x|^{p_{k}{ }^{-1}} e^{-a \Psi_{k}(x)} & x \in \mathfrak{A}_{k} \\ 0 & x \notin \mathfrak{O}_{k}\end{cases}
$$

where $p_{k}$ and $A_{k}$ are given in $\left(\mathrm{A}_{2}\right)$, are p.d.f.-s.

Theorem. - The necessary and sufficient condition for the r.v. $s x_{k}(k=0$, $1, \ldots, n)$ to be distributed according to the p.d.f.-s (4) is that

(C) $y$ and $\left(y_{1}, \ldots, y_{n}\right)$ are independent.

Proof. - The $(n+1)$-dimensional r.v. $\left(y, y_{1}, \ldots, y_{n}\right)$ has its p.d.f. $g\left(y, y_{1}, \ldots, y_{n}\right)$ given on $\mathfrak{B}^{*}=\mathfrak{B}_{0} \times \mathfrak{B}$ by the formula

$$
g\left(y, y_{1}, \ldots, y_{n}\right)=f_{0}\left(x_{0}\right) \cdot f_{1}\left(x_{1}\right) \ldots f_{n}\left(x_{n}\right) \cdot|J|
$$


where $\left(y, y_{1}, \ldots, y_{n}\right)$ on $\mathfrak{B}^{*}$ and $\left(x_{0}, x_{1}, \ldots, x_{n}\right)$ on $\mathfrak{A}_{0} \times \mathfrak{A}_{1} \times \ldots \times \mathfrak{C l}_{n}$ are connected by (2), and where the JACOBIAN of (2) is

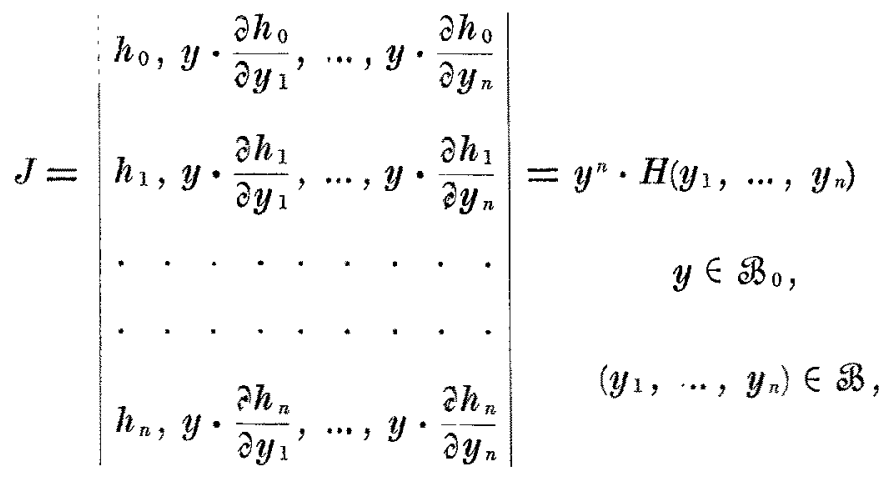

where the function $H$ depends only on $\left(y_{1}, \ldots, y_{n}\right)$ but not on $y$. From the other hand, becanse of the condition $(C)$ the p.d.f. of $\left(y, y_{1}, \ldots, y_{n}\right)$ may be written in form

$$
\begin{array}{r}
g\left(y, y_{1}, \ldots, y_{n}\right)=G_{0}(y) \cdot G\left(y_{1}, \ldots, y_{n}\right) \quad y \in \mathscr{B}_{0} \\
\left(y_{1}, \ldots, y_{n}\right) \in \mathfrak{B}
\end{array}
$$

where $G_{0}$ and $G$ are the p.d.f.-s of $y$ and $\left(y_{1}, \ldots, y_{n}\right)$ respectively.

Becanse the left hand sides of (5) and (7) are equal, then the right hand sides are equal as well, and we may write the following functional equation

$$
\begin{array}{r}
f_{0}\left(x_{0}\right) f_{1}\left(x_{1}\right) \cdot \ldots \cdot f_{n}\left(x_{n}\right)=\gamma_{0}(y) \cdot \gamma\left(y_{1}, \ldots, y_{n}\right) \\
x_{k} \in \mathfrak{A}_{k}, y \in \mathfrak{B}_{0},\left(y_{1}, \ldots, y_{n}\right) \in \mathfrak{B}
\end{array}
$$

where $f_{k}$ are unknown functions satisfying the conditions $\left(\mathrm{A}_{1}\right),\left(\mathrm{A}_{2}\right)$, and where $\gamma_{0}$ and $\gamma$ are given by

$$
\left\{\begin{array}{l}
\gamma_{0}(y)=\frac{G_{0}(y)}{y^{n}}, y \in \mathfrak{B}_{0}, \\
\gamma\left(y_{1}, \ldots, y_{n}\right)=\frac{G\left(y_{1}, \ldots, y_{n}\right)}{H\left(y_{1}, \ldots, y_{n}\right)},\left(y_{1}, \ldots, y_{n}\right) \in \mathfrak{B} .
\end{array}\right.
$$


In order to solve the equation (8) let us divide both its sides by $\prod_{k=0}^{n}\left|x_{k}\right|^{p_{k}-1}$; using (2) we get

where

$$
\begin{array}{r}
\varphi_{0}\left(x_{0}\right) \cdot \varphi_{1}\left(x_{1}\right) \cdot \ldots \cdot \varphi_{n}\left(x_{n}\right)=\gamma_{0}^{*}(y) \cdot \gamma^{*}\left(y_{1}, \ldots, y_{n}\right) \\
x_{k} \in \mathfrak{A}_{k}, y \in \mathfrak{B}_{0}\left(y_{1}, \ldots, y_{n}\right) \in \mathfrak{B}
\end{array}
$$

(11)

$$
\left\{\begin{array}{l}
\varphi_{k}{ }_{k}^{\prime}(x)=\frac{f_{k}(x)}{|x|^{p_{k}-1}}, \quad x \in \mathfrak{Q}_{k} \\
\gamma_{0}^{*}(y)=\frac{\gamma_{0}(y)}{y_{k=0}^{\sum_{n}^{n} p_{k}-n-1}}, \quad y \in \mathfrak{B}_{0} \\
\gamma^{*}\left(y_{1}, \ldots, y_{n}\right)=\frac{\gamma\left(y_{1}, \ldots, y_{n}\right)}{\prod_{k=0}^{n}\left|h_{k}\left(y_{1}, \ldots, y_{n}\right)\right|^{p_{k}-1}}\left(y_{1}, \ldots, y_{n}\right) \in \mathfrak{B}
\end{array}\right.
$$

Putting $y \rightarrow 0$ and using $\left(\mathrm{B}_{1}\right),\left(\mathrm{B}_{2}\right)$ and $\left(\mathrm{B}_{3}\right)$ we get also $x_{k} \rightarrow 0$. Putting this into (10) we get

$$
\varphi_{0}(0) \cdot \varphi_{1}(0) \cdot \ldots \cdot \varphi_{n}(0)=\gamma_{\hat{o}}^{*}(0) \cdot \gamma_{1}\left(y_{1}, \ldots, y_{n}\right) \quad\left(y_{1}, \ldots, y_{n}\right) \in \mathfrak{B}
$$

where according to (1) and (11)

$$
\begin{cases}\varphi_{k}(0)=\lim _{x \rightarrow 0} \varphi_{k}(x)=\lim _{x \rightarrow 0} \frac{f_{k}(x)}{\left.|x|\right|_{k}-1}=A_{k}, & x \in \mathfrak{A}_{k} \\ \gamma_{0}^{*}(0)=\lim _{y \rightarrow 0} \gamma^{*}(y) & y \in \mathscr{B}_{0} .\end{cases}
$$

Dividing sides by sides (10) and (12) we get

$$
\varphi_{0}^{* *}\left(x_{0}\right) \cdot \varphi_{1}^{*}\left(x_{1}\right) \cdot \ldots \cdot \varphi_{n}^{*}\left(x_{n}\right)=\gamma_{0}^{* * *}(y) \quad x_{k} \in \mathfrak{A}_{k}, \quad y \in \mathfrak{B}_{0}
$$

where

$$
\begin{cases}\varphi_{k}^{*}\left(x_{k}\right)=\frac{\varphi_{k}\left(x_{k}\right)}{\varphi_{k}^{\prime}(0)}, & x_{k} \in \mathfrak{A}_{k} \\ \gamma_{0}^{* *}(y)=\frac{\gamma_{0}^{*}(y)}{\gamma_{0}^{*}(0)} & y \in \mathfrak{B}_{0} .\end{cases}
$$


Let us put

$$
\begin{cases}u_{k}=\Psi_{k}\left(x_{k}\right), \varphi_{k}^{*}\left(x_{k}\right)=\lambda_{k}\left(\Psi_{k}\left(x_{k}\right)\right) & x_{k} \in \mathfrak{A}_{k}, u_{k} \in \mathfrak{C} \\ v=\Psi(y), \quad \gamma_{0}^{* *}(y)=\lambda(\Psi(y)) & y \in \mathfrak{B}_{0}, v \in \mathfrak{C}\end{cases}
$$

then the equation (14) becomes

$$
\lambda_{0}\left(u_{0}\right) \cdot \lambda_{1}\left(u_{1}\right) \cdot \ldots, \lambda_{n}\left(u_{n}\right)=\lambda(v), \quad u_{k} \in \mathbb{C}, v \in \mathbb{C} .
$$

Putting (16) into $\left(\mathrm{B}_{3}\right)$ we get it as

$$
v=u_{0}+u_{1}+\ldots+u_{n}, \quad u_{k} \in \mathcal{C}, v \in \bigodot
$$

that is why the equation (17) meets the form

$$
\prod_{k=0}^{n} \lambda_{k}\left(\boldsymbol{u}_{k}\right)=\lambda\left(\sum_{k=0}^{n} \boldsymbol{u}_{k}\right) \quad \boldsymbol{u}_{k} \in \mathcal{C}
$$

where $\lambda_{k}$ are continuous and $\lambda_{k}(0)=1$. This is the known Pexider equation (see [1], p. 141). Putting $u_{j} \rightarrow 0$ for $j \neq k$ we get

$$
\lambda_{k}\left(u_{k}\right)=\lambda\left(u_{k}\right) \quad u_{k} \in \mathcal{C} .
$$

Putting (20) into (19) we get the equation

$$
\prod_{k=0}^{n} \lambda\left(\boldsymbol{u}_{k}\right)=\lambda\left(\sum_{k=0}^{n} \boldsymbol{u}_{k}\right) \quad \boldsymbol{u}_{k} \in \mathfrak{C}
$$

for the only function $\lambda$ on $\mathcal{C}$, continuous, and for which $\lambda(0)=1$. This is the known CaUOHY equation (see [1], p. 31).

Its solution is

$$
\lambda(u)=e^{-a u} \quad u \in \mathfrak{C}
$$

where $a$ is a real constant. Putting (22) into (20) we get

$$
\lambda_{k}(u)=e^{-a \omega} \quad u \in \mathfrak{C} .
$$

Putting (23) into (16) we get

$$
\varphi_{k}^{*}(x)=e^{-a \Psi_{k}(x)} \quad x \in \mathfrak{A}_{k} .
$$

Putting (24) into (15) we get

$$
\varphi_{k}(x)=A_{k} \cdot e^{-a \Psi_{k}(x)} \quad x \in \mathfrak{E}_{h} .
$$


Putting (25) into (11) we get

$$
f_{k}(x)=A_{k}|x|^{p_{k}-1} e^{-a \Psi_{k}(x)} \quad x \in \mathfrak{Q}_{k} .
$$

This ends the proof of the sufficient condition of the theorem.

The proof of the necessary condition of the theorem is obvious by substitution (4) and (6) into (5), using (2) and noting that the right side of (5) becomes the right side of (7).

Example 1. - Pat

$$
A_{k}=(-\infty, 0) \cup(0, \infty), p_{k}=1,(k=0,1, \ldots, n), a=\frac{1}{2 \sigma^{2}}(\sigma>0),
$$

$$
\left(\begin{array}{lr}
x_{0}=y \sin y_{1} & y \in \mathfrak{B}_{0}, \\
x_{1}=y \cos y_{1} \sin y_{2} & \left(y_{1}, \ldots, y_{n}\right) \in \mathfrak{B} \text { where } \\
x_{2}=y \cos y_{1} \cos y_{2} \sin y_{3} & \mathscr{B}=\left(\left(y_{1}, \ldots y_{n}\right) ; \frac{-\pi}{2}<y_{k}<\frac{\pi}{2},\right.
\end{array}\right.
$$$$
\left.k=1,2, \ldots, n-1,0<y_{n}<2 \pi\right\}
$$

$x_{n-1}=y \cos y_{1} \cos y_{2} \ldots \cos y_{n-1} \sin y_{n}$

$x_{n}=y \cos y_{1} \cos y_{2} \ldots \cos y_{n-1} \cos y_{n}$

We see that the condition $\left(\mathrm{B}_{3}\right)$ becomes $x_{0}^{2}+x_{1}^{2}+\ldots+x_{n}^{2}=y^{2}$. That is why $\Psi(y)=y^{2}, \Psi_{k}\left(x_{k}\right)=x_{k}^{2}$.

The independency of $y$ and $\left(y_{1}, \ldots, y_{n}\right)$ is the necessary and sufficient condition for $x_{k}$ to be normally distributed with zero mean and common standard deviation (see TAMHANKAR [3]).

Ex.MPLe 2. - Put

$$
\begin{gathered}
\mathfrak{A}_{k}=(-\infty, 0) \cup(0, \infty), p_{k}=1, \Psi(y)=\log \left(1+y^{2}\right), \\
\Psi_{k}\left(x_{k}\right)=\log \left(1+x_{k}^{2}\right)(k=0,1, \ldots, n) .
\end{gathered}
$$

Then we get the characterization of the generalized CaUCHY distribution given by the p.d.f.

$$
f(x)=\frac{A}{\left(1+x^{2}\right)^{a}}, \quad x \in \mathfrak{Q}_{k}
$$

where $a$ is a positive constant. 
Example 3. - Pat

$$
\begin{aligned}
& \mathfrak{A}_{k}=(0, \infty), k=0,1, \ldots, n, \\
& x_{0}=y\left(1-y_{1}-\ldots-y_{n}\right) \quad y \in \mathfrak{B}_{0} \\
& x_{1}=y y_{1} \quad\left(y_{1}, \ldots, y_{n}\right) \in \mathfrak{B} \text { where } \\
& x_{2}=y y_{2} \quad \mathfrak{B}=\left\{\left(y_{1}, \ldots, y_{n}\right) ; y_{k}>0, k=1, \ldots, n,\right. \\
& \left\{\begin{array}{l}
\cdot \cdot \cdot \\
\cdot \cdot \cdot \\
x_{n}=y y_{n}
\end{array}\right. \\
& \left.y_{1}+\ldots+y_{n}<1\right\}
\end{aligned}
$$

We see that the condition $\left(\mathrm{B}_{3}\right)$ becomes $y=x_{0}+x_{1} \ldots+x_{n}$. That is why $\Psi(y)=y, \Psi_{k}\left(x_{k}\right)=x_{k}$. The independency of $y$ and $\left(y_{1}, \ldots, y_{n}\right)$ is the neces. sary and sufficient condition for $x_{k}$ to have the gamma distribution given by the density

$$
f_{k}\left(x_{k}\right)=\left\{\begin{array}{lr}
\frac{a_{k}^{p_{k}}}{\Gamma\left(p_{k}\right)} x_{k_{k}}^{p_{k}-1} e^{-a x_{k}} & \text { for } x_{k} \in \mathfrak{d}_{n} \\
0 & \text { otherwise }
\end{array}\right.
$$

where $a$ and $p_{k}$ are positive constants. (See KonLanskI [2]).

Example 4. - Put

$$
\mathfrak{A}_{k}=(0, \infty), \Psi(y)=\log (1+y), \Psi_{k}^{\prime}(x)=\log (1+x), k=0,1, \ldots, n
$$

Then we get the characterization of the beta distribution of the second kind given by the density.

$$
f_{k}(x)=\left\{\begin{array}{lr}
\frac{1}{B\left(p_{k}, a-p_{k}\right)} \frac{x^{p_{k}-1}}{(1+x)^{a}} & x \in \mathfrak{A}_{k} \\
0 & \text { otherwise }
\end{array}\right.
$$

where $p_{k}>0$ and $a>\max \left(p_{0}, p_{1}, \ldots, p_{n}\right)$. 


\section{REFERENOES}

[1] J. AcZÉL, Lectures on functional equations and their applications, Academic Press New York and London 1966.

[2] I. I. KoTlansis, Una caratterizanione della distribuzione gamma per mezzo di stati. stiche indipendenti, Rendiconti di matematica e delle sue applicarioni, 1969, (3-4) Vol. 2, Serie VI, pp. 1-5.

[3] M. V. TAmhankar, A characterization of normality, The Annals of Mathematical Statistics, Vol. 38 No. 6, December 1967, pp. 1924-1927. 